\title{
PRODUÇÃO E CONSUMO DE FRUTAS NO JAPÃO
}

\author{
André Freire $\mathrm{Cruz}^{1}$, Ivone Midori Icuma ${ }^{2}$, Osvaldo Kiyoshi Yamanishi ${ }^{2}$ \\ ${ }^{1}$ Kyoto Prefectural University, Graduate School of Life and Environmental Sciences, 1-5 Shimogamohangi-cho, Sakyo-ku, Kyoto \\ 606-8522, Japan, e-mail: andre@kpu.ac.jp \\ ${ }^{2}$ Universidade de Brasília, Faculdade de Agronomia e Medicina Veterinária, Campus Universitário Darcy Ribeiro, Asa Norte, \\ 70919-970 - Brasília, DF - Brasil - Caixa-Postal: 04508
}

\section{RESUMO}

O Japão é um país que historicamente tem produzido frutas, sempre visando a qualidade, especialmente a boa aparência, uniformidade e sabor e é hábito dos consumidores locais pagarem caro por produtos de alta qualidade. O manejo, que vai desde a escolha da cultivar apropriada até a comercialização, é sempre focalizado para atender estas exigências, e por isso os frutos produzidos localmente são relativamente caros comparados com outros países. Para todas as fruteiras existe sempre uma cultivar que é de preferência e que se destaca com mais de $40 \%$ da produção local. Tanto a área plantada como a produção têm diminuído para a maioria das frutas. Este artigo trata da produção, consumo de frutas no Japão, bem como uma apresentação generalizada sobre o manejo das principais fruteiras produzidas localmente.

Palavras-chave: Frutas do Japão, comercialização, pomares, qualidade

\section{PRODUCTION AND CONSUMPTION OF FRUITS IN JAPAN}

\begin{abstract}
Japan is a country which has a history to produce fruits always focusing on the quality, especially the external appearance, uniformity and taste and it is common to pay high prices for products of high quality. The management of orchards, from the cultivar selection to the trading, always follows these requirements, thus the local fruits produced are very expensive as compared to other countries. For all kind of fruits there always exist a variety that has preference of consumers and composes more than $40 \%$ of the local production. The production and the planted area have decreased for the majority of the fruits. This paper describes the production and consumption of fruits in Japan. A general introduction of the management of main fruits locally produced is discussed as well.
\end{abstract}

Key words: Japan fruits, trading, orchard, quality

\section{INTRODUÇÃO}

Recentemente, sementes de castanha (Castanea sativa) foram escavadas de ruínas de áreas ecúmenas no norte de Japão, onde habitavam pessoas há milhares de anos atrás. Uma investigação mais profunda indicou que estas castanheiras eram cultivadas e não plantas nativas, devido a sua composição genotípica similar. Muitas fruteiras, como o citros, foram trazidas da China, Coreia e Sudeste Asiático antes da formação de um país unificado no século 5. De acordo com a literatura "A Record of Ancient Matters" escrito em 712, um nobre fez visitas a outros países e trouxe de volta ao Japão Citrus 
tachibana. Cerejeiras (Prunus serrulata) também têm sido plantadas em pátios de templos e florestas, mas se tornaram bem populares, no século 17 onde pessoas se divertiam com suas floradas que marcam a chegada da primavera (Sugiyama, 2006).

Durante a era Edo (1603 a 1868) o Japão foi marcado por sua prosperidade, onde interesse pela horticultura se espalhou em todas as classes da sociedade. A cidade de Edo (Atual Tóquio) se tornou um grande centro consumidor de produtos hortícolas. No caso específico de fruteiras, somente citros e uva eram transportados para cidades remotas, enquanto outras eram cultivadas em vilarejos ao redor de grandes cidades para consumo próprio. No final dos anos 50, o Japão concentrou investimento na indústria hortícola e no cultivo em casas de vegetação, principalmente visando à qualidade dos frutos e a proteção a pragas e doenças. No entanto, nos anos 80 em função de constantes oscilações no mercado, houve um crescimento nas importações resultando numa queda geral dos preços (Sugiyama, 2006).

\section{A QUESTÃO DA QUALIDADE}

Os produtores japoneses têm sempre
dado importância à qualidade, principalmente de frutas. Isto se dá porque o consumidor japonês tem continuamente demonstrado interesse em pagar caro por produtos de alta qualidade, já que o aspecto visual é algo muito importante na cultura local. Outro fator importante para a questão dos hábitos locais estarem no apelo pela constituição nutricional do alimento, que esteja associado a hábitos saudáveis. Ainda considerando os aspectos culturais envolvidos no consumo de frutas, é necessário levar em conta que a entrada de alguns produtos deveria estar acompanhada de campanhas que induzam mudanças de hábitos da população consumidora. Um bom exemplo disso são os caquis, consumidos em associação com as festas tradicionais de família no início de cada ano (ACN, 2002). Atualmente, os pesquisadores têm visado lançar cultivares de alta qualidade e ao mesmo tempo sugerir manejos que venham diminuir a mão-de-obra e minimizar o impacto ambiental (Sugiyama, 2006).

\section{APRESENTAÇÃO GENERALIZADA DE CADA FRUTEIRA}

Em seguida está a caracterização das principais frutas produzidas no Japão, sendo que suas principais cultivares estão mencionadas na tabela 1 . Para cada fruteira existem 1 ou 2 cultivares (em negrito) que ocupam mais de $50 \%$ da área plantada, por terem preferência do consumidor em termos de qualidade externa e interna. Entretanto, estas nem sempre estão com o preço mais em conta no varejo, ex: uva "Delaware" e maçã "Fuji". Para cada cultivar, mesmo sendo prioritária, caso atinja uma situação de superprodução, o governo faz um programa para estimular os produtores a partirem para cultivares alternativas. É neste ponto que se ressalta a importância de manter outras cultivares, ex: uva "Fujiminori".

\section{Ameixa (Prunus salicina):}

A cultivar japonesa da ameixa ( $P$. salicina) é originária da China e veio ao Japão a cerca de 2000 anos. Seu cultivo tem crescido após o cruzamento com ameixa americana ( $P$. americana), a partir daí surgiram variedades como "Beauty", "Santa rosa", etc. Os principais produtores são as prefeituras de Yamanashi, Wakayama e Nagano. Atualmente, a ameixa é considerada um alimento saudável devido a sua riqueza em fibras, potássio e caroteno (Japan-Guide, 2010). A cultivar "Oishiwase sumomo" se destaca entre as outras (Tabela 1). 
Tabela 1. Relação de fruteiras produzidas no Japão e suas principais cultivares em destaque.

\begin{tabular}{|c|c|c|}
\hline Fruteira & Cultivar & Fonte \\
\hline $\begin{array}{l}\text { Ameixa } \\
(\text { Prunus salicina })\end{array}$ & $\begin{array}{l}\text { Beauty, Methley, Oishiwase sumomo e } \\
\text { Santa Rosa }\end{array}$ & (Kubota, 2006) \\
\hline $\begin{array}{l}\text { Apricota japonesa } \\
\text { (Prunus mume) }\end{array}$ & Baigou, Gojiro, Ryukyo koume e Nanko & (Kubota, 2006) \\
\hline $\begin{array}{l}\text { Caqui } \\
\text { (Diospyros kaki) }\end{array}$ & $\begin{array}{l}\text { Fuyu, Hiratanenashi, Jiro, Saijo, Soshu e } \\
\text { Tonewase }\end{array}$ & (Yamada, 2006) \\
\hline $\begin{array}{l}\text { Castanha } \\
\text { (Castanea sativa) }\end{array}$ & Ginyose, Ishizuchi, Tanzawa e Tsukuba & (Sawamura, 2006) \\
\hline $\begin{array}{l}\text { Cereja } \\
\text { (Prunus serrulata) }\end{array}$ & $\begin{array}{l}\text { Hokko, Napoleón, Satou nishiki e } \\
\text { Takasago, }\end{array}$ & (Kubota, 2006) \\
\hline $\begin{array}{l}\text { Citros } \\
\text { (Citrus unshiu) }\end{array}$ & $\begin{array}{l}\text { Hassaku, Iyo tangor, Satsuma mandarin, } \\
\text { Natsudaidai e Ponkan }\end{array}$ & (Mizutani, 2006) \\
\hline $\begin{array}{l}\text { Figo } \\
\text { (Ficus carica) }\end{array}$ & Houraishi e Masui dauphine & (Manago, 2006) \\
\hline $\begin{array}{l}\text { Kiwi } \\
\text { (Actinidia chinensis) }\end{array}$ & Kosui, Sanuki Gold, Sanryoku e Hayward & (Yakushiji, 2006) \\
\hline $\begin{array}{l}\text { Maçã } \\
\text { (Malus domestica) }\end{array}$ & $\begin{array}{l}\text { Fuji, Jonagold, Orin, Mutsu, Sansa, } \\
\text { Sekaiichi, Tsugaru e Yoko }\end{array}$ & (Arakawa \& Komori, 2006) \\
\hline $\begin{array}{l}\text { Pêra japonesa } \\
\text { (Pyrus pyrifolia) }\end{array}$ & Hosui, Kosui, Nijisseiki e Niitaka & (Tamura, 2006) \\
\hline $\begin{array}{l}\text { Pêssego } \\
\text { (Prunus persica) }\end{array}$ & $\begin{array}{l}\text { Hakuho, Akatsuki, Gyousei, Okubo e } \\
\text { Chikuma }\end{array}$ & (Kubota, 2006) \\
\hline $\begin{array}{l}\text { Uva } \\
\text { (Vitis vinifera) }\end{array}$ & $\begin{array}{l}\text { Campbell Early, Delaware, Gorby, } \\
\text { Kyoho, Muscat Bailey A, Suiho, Pione }\end{array}$ & (Shiraishi, 2006) \\
\hline
\end{tabular}

\section{Apricota japonesa (Ume) (Prunus mume):}

Botanicamente o fruto da apricota japonesa é semelhante ao damasco. É mais consumida em conserva (umeboshi) e posta no meio de uma tigela de arroz branco ou dentro de bolos de arroz (onigiri). Também são utilizados para produzir umeshu (vinho de apricota), um licor doce misturado com o sake (nihonshu) e açúcar.

Ao contrário das cerejeiras floridas, a Apricoteira japonesa florida do Japão produz os frutos comestíveis. O cultivo destas árvores é originário da China, e foi introduzida no Japão na época que o umeboshi já estava sendo consumido durante o período Nara (710-784) (Japan-Guide, 2010). A cultivar "Nanko" possui a maior área plantada (Tabela 1).
Caqui (Diospyros kaki):

São geralmente consumidos in natura depois de descascados e cortados em pedaços, assim como maçãs e pêra japonesa. Também são consumidos secos, semelhante ao figo. A safra se concentra no final do outono e inverno, quando para algumas variedades como "hiratanenashi", destacada no mercado local (Tabela 1), são submetidos a remoção da adstringência pela desidratação, etanol ou $\mathrm{CO}_{2}$ após a colheita (Yamada, 2006).

Originário da China, o caqui é cultivado no Japão desde o século 7 (JapanGuide, 2010). 


\section{Castanha (Nihon Kuri) (Castanea sativa):}

O cultivo da castanha ( $C$. sativa) tem uma longa história no Japão, onde se tornou uma grande fonte alimentícia. Documentos históricos, como um escrito antigo do "Nihonshoki" do imperador Jito (693 d.C), onde este ordenou o cultivo de algumas frutas incluindo a castanha para saciar a fome no país.

O programa de melhoramento da National Institute of Fruit Tree Science: NIFTS sempre visa lançar uma variedade com boa produtividade, tamanho grande e casca facilmente removível (Japan-Guide, 2010). A cultivar "Tsukuba" ocupa a maior área plantada (Sawamura, 2006).

\section{Cereja (Prunus serrulata):}

A cereja ( $P$. serrulata) foi introduzida no Japão no início da era Meiji (1868-1912). A cultivar "Satou Nishiki" foi plantada no início de 1900 e nomeado em homenagem a seu cultivador, Sr. Sato Esuke, sendo esta de coloração avermelhada a mais popular. $\mathrm{Na}$ prefeitura de Yamagata, principal produtora, o fruto é celebrada como um símbolo local (Japan-Guide, 2010). A mesma é a principal cultivar consumida no Japão (Kubota, 2006).

\section{Citros (Unshu Mikan) (Citrus unshiu):}

A tangerina (mikan) (Citrus unshiu) é a fruta cítrica mais consumida entre os japoneses (Tabela 1). Possui uma casca facilmente removível e não tem sementes e é muito popular como sobremesa ou lanche na época de safra, no início do inverno. A tangerina foi trazida ao Japão da China, a cerca de 400 anos, de onde foi levada ao Ocidente e se tornou bem conhecida. Mundialmente o Japão é um país destacado na produção desta fruta, que similarmente a maçã, é uma das poucas frutas japonesas que são exportadas em quantidades consideráveis. As prefeituras de Wakayama,
Ehime e Shizuoka são as principais produtoras (Japan-Guide, 2010).

Figo (Ficus carica):

São mais consumidos descascados e in natura, mas também são muito populares na forma seca ou em compotas. $F$. carica foi introduzido da China no Japão no início do século 17, quando seu fruto também era conhecido como "caqui estrangeira" (JapanGuide, 2010).

As cultivares "Dauphine Masui" e "Houraishi" são as únicas para plantio comercial. A "Dauphine Masui" que é relativamente grande, cujo nome é em homenagem ao o Sr. Masui que a importou da Califórnia (Manago, 2006) e cultivou no início do século 20 (Japan-Guide, 2010).

\section{Kiwi (Actinidia chinensis):}

Diferente das cultivares vendidas em outros países, o fruto de A. chinensis é composto de uma casca fina, peluda e parte interna comestível suculenta e verde. A safra começa no início do inverno, mas kiwi importado se encontra disponível durante todo o ano, o que constitui na sua maioria, sendo que a Nova Zelândia é o principal país exportador para o Japão (Japan-Guide, 2010).

A cultivar "Hayward" originaria da Nova Zelândia é a mais comercializada e ocupa 90\% dos pomares no Japão (Yakushiji, 2006).

\section{Maçã (Malus domestica):}

Seu cultivo generalizado iniciou-se na Era Meiji (1868-1912) e hoje é uma das frutas mais populares no Japão, e uma das poucas frutas que são exportadas. No Japão, os frutos de $M$. domestica são geralmente consumidos descascados e in natura, cuja colheita é entre o outono e início do inverno. Entre todas as variedades, a "Fuji" é a mais 
popular devido a seu tamanho grande e cor vermelha (Arakawa \& Komori, 2006).

Acredita-se de que o nome está associado a pequena cidade da prefeitura de Aomori, onde foi cultivada em 1930. Atualmente esta é a maior produtora de maçãs do Japão, sendo que a cidade de Hirosaki, em particular, está intimamente associada com sua produção (Japan-Guide, 2010).

\section{Pêra (Pyrus pyrifolia):}

Existem a pêra ocidental e a pêra japonêsa (nashi), com algumas diferenças entre estas. Comparado com a pêra ocidental, a japonêsa é maior, mais crocante, mais leve, com uma casca mais espessa e formato esférico. A pêra japonêsa está disponível durante o verão e outono e geralmente é consumida descascada. Tem sido cultivada localmente desde os primórdios da civilização japonesa. As cultivares "Kosui" e "Hosui" ocupam mais de $50 \%$ do total da área plantada (Tamura, 2006). As ocidentais também estão disponíveis no país, e são conhecidas como yonashi (pêras ocidentais) (Japan-Guide, 2010).

\section{Pêssego (Prunus persica):}

As cultivares de pêssegos japonesas são geralmente maiores, mais macias e mais caras do que as ocidentais, o mesocarpo é geralmente branco e as vezes amarelo. $\mathrm{O}$ período de safra chega no verão (JapanGuide, 2010). As cultivares em destaque são "Hakuho" e "Akatsuki" com maior área plantada (Kubota, 2006). Os pêssegos são provenientes da China, no período Yayoi (300 aC-300 dC) e atualmente as prefeituras de Yamanashi e Fukushima são as maiores produtoras (Japan-Guide, 2010).

\section{Uva (Vitis vinifera):}

Como os morangos, as uvas japonesas cujas variedades são grandes, doces e caras, são as preferidas. A principal diferença é que a pele das uvas japonesas é geralmente muito espessa e por isso são descascadas antes do consumo (Japan-Guide, 2010). Uma das variedades mais populares é a uva escura "Kyoho", que é grande, a "Delaware" ocupa a segunda posição em termos de área plantada (Shiraishi, 2006). Semelhante as outras fruteiras as uvas foram inicialmente introduzidas a partir da China em torno do Período Nara (710-784). Mais recentemente novas variedades chegaram do Ocidente no final de 1800 (Japan-Guide, 2010).

\section{CARACTERÍSTICAS DO MANEJO E PRODUÇÃO}

Existe uma grande variedade de frutas produzidas no Japão com suas respectivas cultivares (Tabela 1), muitas das quais foram trazidas do exterior e localmente são bem reconhecida, enquanto outras são nativas do Japão e não tão bem conhecidas além de países da Ásia Oriental. A colheita de frutas tornou-se uma das atrações turísticas e também um negócio bem sucedido nos últimos anos (Japan-Guide, 2010).

A produção comercial de frutos se elevou na era Edo, principalmente a de citros, cujas variedades foram sendo substituídas por "Satsuma mandarin" na era Meiji (Mizutani, 2006; Sugiyama, 2006).

Já as maçãs são consideradas as de maior popularidade no Japão atingindo um total de 798.200 toneladas em 2010, a segunda maior produção depois dos citros (FAOSTAT, 2012).

Quanto ao manejo, dependendo da fruteira, começa com a escolha do portaenxerto apropriado para o local, cuja compatibilidade com o enxerto, resistência a fatores de solo, dependendo da região e da salinidade, é fator crucial. 
Outras atividades fazem parte da construção e manutenção do pomar como espaçamento, fertilização, poda, desbaste de frutos visando produzir frutos maiores, controle severo de pragas e doenças, ensacamento, anelamento e para algumas fruteiras como videira e tangerina o cultivo em casa-de-vegetação. No caso de videiras, macieiras, pêra e kiwi é necessária a construção de suportes também.

As videiras são geralmente cultivadas em sistema de latada e em estufa para evitar problemas com tufão e doenças fúngicas e melhor aproveitamento da luz solar (Shiraishi, 2006).

Os dados sobre a produção, área plantada e produtividade encontram-se na Tabela 2.

A produção de frutas no Japão vem diminuindo num período de 4 anos, mesmo com um aumento entre 2006 e 2008, exceto a uva, para algumas fruteiras. Já para a cereja houve um pequeno aumento entre 2008 e 2010. Também frutas tropicais são produzidas localmente como mamão, abacaxi e banana, porém em quantidades relativamente baixas ou quase insignificantes comparadas com as temperadas.

Similaridade ocorreu para a área plantada na qual, deixando a cereja a parte, houve um decréscimo para todas as fruteiras. No caso de citros houve um pico na produção e área plantada em 1975 e em 1994 eram de 1.804 .750 toneladas e 108.182 ha respectivamente (Yamanishi \& Cruz, 2001), ou seja, quase o dobro da atual.

Apesar de haver uma diminuição na área plantada para algumas fruteiras, a produção aumentou entre 2006 e 2008 levando a entender que houve um aumento de produtividade neste intervalo. Talvez isto se deva a um grande investimento em tecnologia, melhoramento visando maior produtividade e/ou substituição por cultivares mais produtivas.

Tabela 2. Produção, área plantada e produtividade de fruteiras no Japão.

\begin{tabular}{|c|c|c|c|c|c|c|c|c|c|}
\hline & \multicolumn{3}{|c|}{$\begin{array}{c}\text { Produção (ton/ano) x } \\
1000\end{array}$} & \multicolumn{3}{|c|}{$\begin{array}{l}\text { Área plantada (ha) } \\
\text { x } 1000\end{array}$} & \multicolumn{3}{|c|}{ Produtividade (ton/ha) } \\
\hline & 2006 & 2008 & 2010 & 2006 & 2008 & 2010 & 2006 & 2008 & 2010 \\
\hline Ameixa- & 21,4 & 26,0 & 20,9 & 3,1 & 3,0 & 3,0 & 6,9 & 8,6 & 7,0 \\
\hline Apricota & 119,7 & 121,0 & 92,4 & 18,0 & 17,4 & 14,0 & 6,7 & 7,0 & 6,6 \\
\hline Castanha & 23,1 & 25,3 & 23,5 & 23,3 & 22,5 & 21,7 & 1,0 & 1,1 & 1,1 \\
\hline Caqui & 232,7 & 266,6 & 189,4 & 23,5 & 23,0 & 22,4 & 9,9 & 11,6 & 8,5 \\
\hline Cereja & 20,8 & 17,0 & 19,7 & 4,5 & 4,5 & 4,5 & 4,6 & 3,8 & 4,4 \\
\hline Citros & $1.047,8$ & $1.090,1$ & 934,1 & 64,0 & 59,0 & 56,3 & 16,4 & 18,5 & 16,6 \\
\hline Figo & 16,0 & 16,4 & 14,0 & 1,2 & 1,2 & 1,1 & 13,3 & 14,3 & 12,7 \\
\hline Maçã & 831,8 & 910,7 & 798,2 & 40,3 & 39,5 & 38,1 & 20,6 & 23,1 & 21,0 \\
\hline Kiwi & 32,5 & 38,4 & 29,9 & 2,5 & 2,4 & 2,3 & 13,2 & 16,1 & 13,0 \\
\hline Pêra & 319,7 & 361,7 & 284,9 & 16,7 & 16,0 & 15,5 & 19,2 & 22,6 & 18,4 \\
\hline Pêssego & 146,3 & 157,3 & 136,7 & 10,3 & 10,1 & 10,0 & 14,2 & 15,6 & 16,7 \\
\hline Uva & 210,5 & 201,0 & 184,8 & 18,9 & 18,4 & 15,5 & 11,1 & 10,9 & 11,9 \\
\hline
\end{tabular}

Fonte: (FAOSTAT, 2012; MAFF, 2012) 
Dentre muitas estas características distinguem a produção de frutas no Brasil, onde o consumidor está mais voltado ao preço do que a aparência. Com excessão dos pomares cujo foco é a exportação, que visam atender as exigências do país importador, como qualidade externa e controle fitossanitário. Talvez, o ponto onde se pode diferenciar a fruticultura no Brasil e no Japão está no fato ser um país exportador e importador respectivamente.

\section{O PERFIL DO CONSUMIDOR NO JAPÃO}

O consumo de frutas em geral no Japão tem aumentado num período de 4 anos, sendo que houve um aumento de 2003 a 2005 com um pequeno decréscimo em 2007. Entre as frutas citadas, apenas o abacaxi e a maçã tiveram um aumento no consumo per capita, as outras se mantiveram estáveis ou diminuíram. Porém estes dados são referentes a situação do país antes da grande crise econômica mundial que afetou a economia local também. Portanto, a partir de 2008 esses números podem ter radicalmente mudados em função desta crise (Tabela 3 ).

Há de se considerar que a variável econômica seja a principal que afeta o consumo de frutas, não deixando de incluir o componente cultural. O governo local tem se preocupado com o baixo nível de autossuficiência na produção interna de alimentos. No caso específico das frutas, verifica-se uma diminuição na produção doméstica, dados os altos custos de produção.

Desde o início da liberalização das importações (em 1989), o perfil do mercado doméstico mudou radicalmente, inclusive no que diz respeito a mudanças importantes nos aspectos culturais relacionados ao consumo de frutas (eliminando-se as sazonalidades) (ACN, 2002).
Numa visão globalizada (Tabela 4) a Itália o Brasil e os EUA são os países que têm o maior consumo per capita de frutas em geral, de modo que ocupam a primeira colocação nesta sequência: Itália (Laranja, Tangerina) e Brasil (Abacaxi). Entre os países citados o Japão ocupa a antepenúltima colocação neste quadro levando em conta as frutas em geral, estando na frente apenas do Quênia e África do Sul. Entretanto, considerando apenas os países do hemisfério norte o Japão se coloca em último lugar neste parâmetro. Entre todas as frutas citadas, exceto o abacaxi, "grape-fruit" e maçã, o Japão está entre os quatro últimos colocados em termos de consumo per capita. As melhores colocações estão para a maçã e "grape-fruit", sendo o terceiro e quarto colocados, respectivamente.

Estes dados confirmam a dificuldade do consumo de frutas no Japão apesar de ser um país do G7, sendo estas um "artigo de luxo" que pesaria muito num orçamento doméstico caso fossem consumidas em largas quantidades.

O estilo de vida local tem mudado radicalmente com uma percentagem da população idosa aumentando (Yamane, 2006).

A demanda por frutas tem diminuído (Tabela 3), pois o consumo per capita de frutas no Japão não tem aumentado em 3 anos. 
Tabela 3. Consumo per capita de frutas no Japão.

\begin{tabular}{lccc}
\hline \hline & & $\mathrm{kg} / \mathrm{hab} / \mathrm{ano}$ \\
\cline { 2 - 4 } & 2003 & 2005 & 2007 \\
\hline Frutas em peral & 55,4 & 60,6 & 58,2 \\
Abacaxi & 1,5 & 1,9 & 1,9 \\
Banana & 6,4 & 7,0 & 6,3 \\
Grape-fruit & 3,6 & 3,2 & 2,8 \\
Laranja/Tangerina & 12,0 & 12,3 & 11,1 \\
Limões/Lima & 0,7 & 0,7 & 0,6 \\
Maçã & 16,6 & 19,6 & 20,2 \\
Uva & 2,3 & 2,3 & 2,4 \\
\hline Fonte (FAOSTAT, 2012) & & & \\
\hline
\end{tabular}

Fonte: (FAOSTAT, 2012)

Tabela 4. Consumo per capita de frutas (kg/hab/ano) no mundo (média de 2 anos - 2006 e 2007).

\begin{tabular}{|c|c|c|c|c|c|c|c|c|c|}
\hline & País & $\begin{array}{c}\text { Frutas } \\
\text { em } \\
\text { geral }\end{array}$ & Abacaxi & $\begin{array}{c}\text { Laranja/ } \\
\text { Tangerina }\end{array}$ & $\begin{array}{l}\text { Limões } \\
\text { / Lima }\end{array}$ & Uva & $\begin{array}{c}\text { Grape- } \\
\text { fruit }\end{array}$ & Banana & Maçã \\
\hline \multirow{7}{*}{ 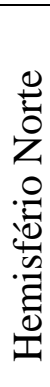 } & China & 63,1 & 0,9 & 12,7 & 0,6 & 3,4 & 0,3 & 5,4 & 13,6 \\
\hline & Egito & 101,0 & 0,0 & 28,5 & 3,5 & 16,1 & 0,0 & 10,1 & 7,1 \\
\hline & EUA & 109,5 & 6,1 & 32,8 & 6,0 & 8,3 & 2,1 & 11,4 & 24,3 \\
\hline & França & 114,1 & 2,8 & 44,8 & 1,8 & 2,9 & 5,1 & 4,2 & 13,6 \\
\hline & Italia & 148,3 & 3,4 & 46,8 & 9,7 & 15,0 & 1,0 & 7,9 & 17,5 \\
\hline & Japão & 56,9 & 1,9 & 10,6 & 0,6 & 2,3 & 2,6 & 6,6 & 19,7 \\
\hline & México & 116,9 & 5,5 & 36,0 & 11,1 & 1,9 & 2,7 & 16,4 & 6,6 \\
\hline \multirow{7}{*}{ 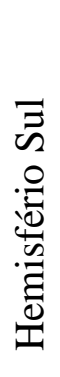 } & Tunísia & 90,6 & 0,1 & 14,0 & 2,6 & 7,4 & 6,4 & 2,8 & 10,2 \\
\hline & África do Sul & 35,1 & 2,3 & 6,4 & 1,4 & 2,2 & 2,6 & 6,7 & 5,4 \\
\hline & Argentina & 66,5 & 0,8 & 21,3 & 6,8 & 1,2 & 2,5 & 11,6 & 16,6 \\
\hline & Australia & 104,3 & 9,6 & 24,1 & 1,8 & 9,9 & 0,5 & 9,1 & 25,2 \\
\hline & Brasil & 109,2 & 10,7 & 32,0 & 3,5 & 4,0 & 0,3 & 30,6 & 4,0 \\
\hline & Nova Zelândia & 108,0 & 4,1 & 22,1 & 1,1 & 0,3 & 0,8 & 18,0 & 7,2 \\
\hline & Quênia & 54,6 & 7,4 & 0,8 & 0,0 & 0,0 & 0,3 & 13,8 & 0,2 \\
\hline
\end{tabular}

Fonte: (FAOSTAT, 2012) 


\section{AGRADECIMENTOS}

Os autores deste artigo gostariam de agradecer aos membros da FAO e Japanguide por autorizarem a utilizar suas informações. Também ao Dr. Akihiro Katsura, Kyoto Prefectural University, pelo auxílio no levantamento de alguns dados para a confecção das tabelas.

\section{REFERÊNCIAS BIBLIOGRÁFICAS}

ACN. 2002. Asamediator Consultoria de Negócios Ltda. Estudo sobre o Mercado de Frutas no Japão para a Embaixada do Brasil em TóquioJapão. São Paulo, 111 p.

ARAKAWA, O.; S. KOMORI. 2006. Apple. In: S. YAZAWA; N. SUGIYAMA, et al (Ed.). Horticulture in Japan. 2006. Kyoto: The Japanese Society for Horticultural Science Shoukadoh Publication, $334 \mathrm{p}$.

FAOSTAT. 2012. Food Agriculture Organization (FAO), Statistical database. Rome: Food Agriculture Organization, 2013.

JAPAN-GUIDE. 2010. Japanese fruits. Disponível em: <http://www.japanguide.com/e/e2347 .html> Acesso em: Junho de 2010.

KUBOTA, N. Stone fruits. 2006. In: S. YAZAWA; N. SUGIYAMA, et al (Ed.). Horticulture in Japan. 2006. Kyoto: The Japanese Society for Horticultural Science - Shoukadoh Publication. 334 p.

MAFF. 2012. The 85th statistical yearbook of Ministry of Agriculture, Forestry and Fisheries. Disponível em: <http://www.maff.go.jp/e/tokei/kikaku/n enji_e/85nenji/index.html> Acesso em: Junho de 2012

MANAGO, N. 2006. In: S. YAZAWA; N. SUGIYAMA, et al (Ed.). Horticulture in Japan. 2006. Kyoto: The Japanese Society for Horticultural Science Shoukadoh Publication, 334 p.
MIZUTANI, F. Citrus. 2006. In: S. YAZAWA; N. SUGIYAMA, et al (Ed.). Horticulture in Japan. 2006. Kyoto: The Japanese Society for Horticultural Science - Shoukadoh Publication, $334 \mathrm{p}$. SAWAMURA, Y. 2006. Other fruit. In: S. YAZAWA; N. SUGIYAMA, et al (Ed.). Horticulture in Japan. 2006. Kyoto: The Japanese Society for Horticultural Science - Shoukadoh Publication, 334 p. SHIRAISHI, M. Grape. 2006. In: S. YAZAWA; N. SUGIYAMA, et al (Ed.). Horticulture in Japan. 2006. Kyoto: The Japanese Society for Horticultural Science - Shoukadoh Publication, $334 \mathrm{p}$.

SUGIYAMA, N. 2006. Brief history of horticulture in Japan. In: S. YAZAWA; N. SUGIYAMA, et al (Ed.). Horticulture in Japan. 2006. Kyoto: The Japanese Society for Horticultural Science - Shoukadoh Publication, 334 p. TAMURA, F. 2006. Pear. In: S. YAZAWA; N. SUGIYAMA, et al (Ed.). Horticulture in Japan. 2006. Kyoto: The Japanese Society for Horticultural Science - Shoukadoh Publication, $334 \mathrm{p}$. YAKUSHIJI, H. Kiwifruit. 2006. In: S. YAZAWA; N. SUGIYAMA, et al (Ed.). Horticulture in Japan. 2006. Kyoto: The Japanese Society for Horticultural Science - Shoukadoh Publication, $334 \mathrm{p}$. YAMADA, M. 2006. Persimmon. In: S. YAZAWA; N. SUGIYAMA, et al (Ed.). Horticulture in Japan. 2006. Kyoto: The Japanese Society for Horticultural Science - Shoukadoh Publication, 334 p.

YAMANE, K. 2006. Marketing system of horticultural crops. In: S. YAZAWA; N. SUGIYAMA, et al (Ed.). Horticulture in Japan. 2006. Kyoto: The Japanese Society for Horticultural Science Shoukadoh Publication, $334 \mathrm{p}$.

YAMANISHI, O. K.; A. F. CRUZ. 2001.

Panorama da citricultura japonesa. Laranja, v.22, n.2, p.299-320. 